\title{
Esfuerzo y decremento de vigilancia en tareas de discriminación sucesiva y simultánea
}

\author{
Manuel J. Blanco \\ Universidad de Santiago de Compostela \\ JUAN R. LAMAS \\ Universidad de La Coruña \\ Antonio A. Alvarez \\ Universidad Nacional a Distancia, Pontevedra
}

\begin{abstract}
Resumen
El objetivo del experimento presentado en este artículo fue probar la bipótesis de que el decremento de sensibilidad en tareas de atención sostenida, ocurre porque los recursos atencionales o esfuerzo que demanda la tarea no pueden matenerse durante un tiempo prolongado. Comparamos dos tareas que diferian en carga de memoria y registramos tres grupos de indices de esfuerzo o gasto atencional: rendimiento en una tarea secundaria concurrente, esfuerzo subjetivo y actividad cardiovascular (IBI medio y amplitud espectral del componente $0.10 \mathrm{~Hz}$ ). En ambas tareas observamos un declive significativo a lo largo del tiempo en eficiencia o ritmo de procesamiento, indicado por la razón entre la precisión y la velocidad. Los datos acerca del esfuerzo subjetivo y el rendimiento en la tarea secundaria no parecen apoyar la hipótesis original. El principal hallazgo fue que la actividad cardiovascular al comienzo de la tarea, medida por la amplitud del componente $0.1 \mathrm{~Hz}$, era un predictor significativo del decremento de eficiencia en ambas tareas de vigilancia. Si esta amplitud es un indice fisiológico fiable de atención, nuestro resultado sugiere que un procesamiento controlado, con esfuerzo, al comienzo de la tarea previene el decremento de vigilancia.
\end{abstract}

Palabras clave: Actividad cardiovascular, Atención, Atención sostenida, Carga de trabajo, Esfuerzo, Psicofisiología cognitiva, Vigilancia.

\section{Abstract}

The aim of the experiment presented in this paper was to test the bypothesis that the sensitivity decrement in vigilance tasks occurs because the attentional resources or effort required by the task cannot, be maintained over a long period of time. Two tasks which differed in memory load were compared on three groups of measures: secondary-task performance, subjective effort and cardiovascular activity (mean IBI and $1 \mathrm{~Hz}$ spectral amplitude). The sensitivity decrement observed in botb tasks was not significant, although a significant decline was observed in both task in efficiency or informationprocessing rate, indexed by the ratio between accuracy and speed. The data related to subjetive effort and secondary-task performance do not appear to support the original bypothesis. The main finding was that the amplitude of the mid-band at the beginning of the task could be used as predictor of the performance decrement. If this amplitude is a reliable physiological index of attention, our result suggests that an effortful processing ta the beginning of the task prevents the vigilance decrement.

Key words: Cardiovascular activity, Attention, Sustained attention, Workload Effort, Cognitive Psychophysiology, Vigilance.

Agradecimientos: Esta investigación fue financiada por la beca PB89-0550 de la Dirección General de Investigación Científica y Técnica. La investigación comenzó mientras el primer autor era Visiting Fellow en el Management Centre de la Universidad de Aston (Birmingham, Gran Bretaña), financiado por una beca de investigación de la Xunta de Galicia. Agradecemos a Gerald Matthews y F. Valle-Inclan sus comentarios en la preparación de este artículo.

Dirección del autor: Manuel J. Blanco. Dpto. de Psicología Social y Básica. Universidad de Santiago. Santiago de Compostela. Tel.: (981) 563100, ext. 2012. 


\section{6}

\section{INTRODUCCION}

Con las tareas de vigilancia se trata de examinar la habilidad de un individuo para sostener su atención en una fuente de información durante un tiempo más o menos prolongado. Estas tareas tienen unas características comunes: a) todas exigen atender a una fuente de información durante un tiempo largo; b) el observador debe informar de señales más o menos simples (p.e. un punto de luz, un tono puro de frecuencia, etc.); $c$ ) estas señales aparecen de modo infrecuente e impredecible mezcladas con estímulos «ruido». El resultado usual en este tipo de tareas es lo que se denomina «función decremento», que es el declive progresivo a lo largo de la sesión de trabajo en el rendimiento del sujeto, medido por el porcentaje de señales detectadas o por algún índice derivado de la teoría de detección de señales (por ejem. plo, $\left.d^{\prime}\right)$. Uno de los objetivos principales que persigue la investigación en este campo es identificar las varibles de tarea que contribuyen a que se produzca este decremento.

Parasuraman y Davies (Davies y Parasuraman, 1982; Parasuraman, 1979; Parasuraman y Davies, 1977) propusieron una taxonomía de tareas en base a cuatro dimensiones: complejidad de la fuente de información, ritmo de estimulación (o razón de evento), modalidad sensorial y tipo de discriminación señal/ruido. Parasuraman y Davies, basándase en un trabajo previo de Fleishman y colaboradores (Fleishman, 1972; ver también Fleishman y Quaintance, 1984), consideraron a la última dimensión — tipo de discriminacióncomo particularmente importante. Parasuraman y Davies distinguen entre tareas de «discriminación sucesiva» y de «discriminación simultánea». En las primeras, la señal se define como un cambio en alguna característica de los estímulos ruido, de forma que el sujeto debe comparar el estímulo con un valor estándar almacenado en la memoria de trabajo, para decidir si es o no señal; un ejemplo de tarea que implica este tipo de discriminación podría ser la detección de un incremento en la intensidad de una luz intermitente. Las tareas de discriminación simultánea, por el contrario, no demandan el uso de la memoria, puesto que sólo la observación del estímulo basta para decidir si es o no señal; un ejemplo podría ser la detección de un tono sinusoidal que aparece sobre un fondo de ruido blanco.

En un estudio taxonómico de varias tareas de vigilancia, Parasuraman y Davies (1977) encontraron que, independientemente de otros factores (p.e. ritmo de estimulación), el decremento de vigilancia aparecía de modo significativo en las tareas de discriminación sucesiva, pero no en las de discriminación simultánea. Esta diferencia fue examinada directamente por Parasuraman (1979) en un experimento en el que comparó varias tareas de vigilancia que diferían en el tipo de discriminación o carga de memoria; sus resultados mostraron que la sensibilidad (indicada por $d^{\prime}$ ) decrecía a lo largo de la sesión en las condiciones de discriminación sucesiva, mientras que permanecía estable en las de discriminación simultánea. Sin embargo, Parasuraman y Mouloua (1987) informaron recientemente de decrementos de sensibilidad en tareas de discriminación simultánea cuando el nivel de discriminación señal/ruido era bajo. En un experimento sobre discriminación de longitudes de líneas, Parasuraman y Mouloua (1987) manipularon el tipo (sucesiva vs. simultánea) y la dificultad de discriminación (alta, moderada y baja). Encontraron decremento de sensibilidad en las tres condiciones de discriminación sucesiva, pero también en la de discriminación 
simultánea cuando las líneas eran difíciles de discriminar (pero no cuando eran moderada o fácilmente discriminables). Con estos datos, Parasuraman y Mouloua (1987) modificaron ligeramente la hipótesis original (Parasuraman, (1979), sugiriendo que el decremento de sensibilidad podría ser determinado por la cantidad total de recursos atencionales que demanda la realización de la tarea, más que simplemente por la carga de memoria que éste impone. Según esta interpretación, la carga de trabajo o cantidad de recursos atencionales sería mayor, en general, en tareas de discriminación sucesiva, pero si la discriminación simultánea es muy difícil, la demanda de recursos atencionales puede aumentar y puede llevar a un decremento de sensibilidad aún en ausencia de una carga de memoria.

Otros investigadores han informado de resultados experimentales que parecen incongruentes con el punto de vista de Parasuraman. Por ejemplo, Wiener, Curry y Faustina (1984) encontraron un efecto facilitador de la carga de trabajo sobre el rendimiento en una tarea de vigilancia visual cuando se pedía a los sujetos que realizasen al mismo tiempo otra tarea visuo-motora. Warm y colaboradores (1984) encontraron también que el decremento de vigilancia podía invertirse si se incrementaba la complejidad de las operaciones cognitivas que se requerían para detectar la señal. Estos autores encontraron decremento en una tarea simple pero no en otra compleja, e interpretaron esto en términos motivacionales: debido a la mayor demanda cognitiva, la tarea compleja podría haber sido más «interesante» para los sujetos que la tarea simple. Los resultados de un experimento reciente de Koelega y colaboradores (1989) tampoco apoyan la hipótesis del «esfuerzo» acerca del decremento de vigilancia. Compararon cuatro tareas de vigilancia que diferían en carga de memoria y en tipo de estimulación, y utilizaron la actividad electrodermal para determinar el esfuerzo del sujeto en cada tarea; sus datos sugieren que el procesamiento con esfuerzo, más que inducir decremento de sensibilidad, previene su ocurrencia.

La teoría de Parasuraman de que el decremento de sensibilidad en tareas de vigilancia ocurre porque los recursos atencionales o esfuerzo requerido por la tarea no puede mantenerse sin descanso a lo largo de la sesión, puede probarse midiendo estos recursos o esfuerzo (Parasuraman, 1985). En este artículo informamos de un experimento diseñado para probar esta interpretación del decremento de vigilancia. Comparamos dos tareas de vigilancia que diferían en el tipo de discriminación: sucesiva o simultánea. La predicción que hace la teoría de Parasuraman (Davies y Parasuraman, 1977; Parasuraman, 1979, 1985; Parasuraman y Mouloua, (1987) es que la tarea que exige discriminación sucesiva, y que por tanto requiere más capacidad de procesamiento, sería más propensa a mostrar decremento de vigilancia. Los términos «carga de trabajo» y «esfuerzo» no se definen aquí. Ambos términos se utilizan aquí indistintamente, para referirnos al modo en que el sujeto afronta la tarea o distribuye sus recursos atencionales.

Utilizamos tres procedimientos para evaluar la carga de trabajo en las dos tareas: procedimiento de la tarea secundaria, evaluación subjetiva y registro de la actividad cardiovascular.

\section{Tarea secundaria}

Parasuraman (1985) empleó el procedimiento de la tarea secundaria para evaluar la carga de trabajo en dos tareas de vigilancia visual que diferían 
en el ritmo de estimulación. La tarea primaria de vigilancia fue similar a la empleada por Nuechterlein y colaboradores (1983). Los sujetos tenían que detectar un dígito objetivo o señal entre un conjunto de dígitos degradados presentados a un ritmo bajo ( 15 dígitos/minuto) o alto ( 30 dígito/minuto). La tarea secundaria consistía en la detección de tonos de $1.000 \mathrm{~Hz}$ que se presentaban al comienzo y al final de la sesión. El tiempo de reacción (TR) a estos tonos se tomó como índice de la carga de trabajo impuesta por la tarea de vigilancia visual. Los resultados indicaron que la sensibilidad permanecía estable durante la tarea de vigilancia con ritmo de estimulación bajo, pero decrecía en la de ritmo de estimulación alto. Además, y esto es lo más importante, los TRs auditivos eran más altos al final de la sesión que al comienzo en la tarea con ritmo de estimulación alto (que mostraba decremento), mientras que eran similares en la condición de ritmo de estimulación bajo (que no mostraba decremento). Este hallazgo parece apoyar la hipótesis de Parasuraman acerca del decremento de vigilancia.

En nuestro experimento, empleamos una tarea de TR a tonos para evaluar la carga impuesta por las dos tareas de vigilancia primarias. Los tonos se presentaban en una de dos posiciones durante el intervalo entre los estímulos primarios: $100-300$ milisegundos o $900-1.100 \mathrm{mseg}$. Si la tarea de discriminación sucesiva demanda operaciones de memoria, tales como el emparejamiento o ajuste y la repetición, entonces deberíamos esperar que los TRs fuesen más largos en esta tarea que en la de discriminación simultánea, que no demanda el uso de la memoria. Además, los TRs a tonos presentados hacia el final de la sesión de vigilancia deberían ser más largos que los TRs a los tonos presentados al principio.

\section{Esfuerzo subjetivo}

La carga mental de trabajo en cada tarea se evaluó con la National Aeronautics and Space Administration Task Load Index o NASA TLX (Hart y Staveland, 1989). Un estudio de Hart y Staveland (1989) ha mostrado que la TLX es sensible a cambios en los niveles de carga de trabajo en diferentes ocupaciones, entre las que se incluyen experimentos de simulación de vuelo y varias tareas cognitivas tradicionales de laboratorio. La TCX proporciona una puntuación de carga total de trabajo basada en un promedio de las evaluaciones que hace el sujeto en seis escalas: demanda mental, demanda física, demanda temporal, rendimiento propio, esfuerzo y frustración. Se aplica en dos partes: primero, se establecen los pesos de los seis factores, pidiendo al sujeto que haga comparaciones por pares; en la segunda parte, los sujetos evalúan en seis escalas de 0 a 100 puntos, una por factor, la carga de trabajo experimentado durante la tarea; los extremos de las escalas son «baja» y «alta», excepto en la de rendimiento que son «bueno» $\mathrm{y}$ «malo». Finalmente, cada evaluación se multiplica por el peso del factor co. rrespondiente, de modo que su impacto sobre la carga total de trabajo refleje la importancia que tiene cada sujeto. En el presente experimento, sólo empleamos como variables dependientes la evaluación ponderada en la escala de esfuerzo y la puntuación de carga total de trabajo, que corresponde a la media de las seis evaluaciones ponderadas.

\section{Actividad cardiovascular.}

Muchos investigadores han buscado correlaciones entre distintas medidas de actividad cardiovascular y el nivel de decremento de vigilancia (ver Davies 
y Parasuraman, 1982; Parasuraman, 1985). Las medidas más empleadas de actividad cardiovascular han sido la media y variabilidad del ritmo cardíaco. En general, las correlaciones entre la media del ritmo cardíaco y el nivel global de rendimiento de vigilancia no son significativas, y no parece existir un patrón claro de cambio de esta medida fisiológica a lo largo del tiempo (p.e. Thackray, Jones y Touchstone, 1974; Carter y Beh, 1989). Por otra parte, se ha encontrado que la variabilidad del ritmo cardíaco se incrementa durante la tarea de vigilancia, pero sólo correlaciona moderadamente con el nivel de rendimiento (p.e. Thackray, Jones y Touchstone, 1974).

Kalsbeek y Ettema (1963) mostraron que la variabilidad del ritmo cardíaco disminuye si se incrementa la carga de trabajo mental. Varios estudios posteriores han confirmado este hallazgo (ver Mulder, 1980). Utilizando el análisis espectral de los intervalos entre pulsos (Inter-Beat-Intervals, IBs), se han encontrado tres componentes de la variabilidad que parecen estar asociados a diferentes mecanismos de control fisiológico: a) una banda de frecuencias bajas (desde 0.02 a $0.06 \mathrm{~Hz}$ ), que parece estar influida por la actividad vasomotora implicada en la regulación de la temperatura corporal; b) una banda de frecuencia medias (desde 0.07 a $0.14 \mathrm{~Hz}$ ), afectada por mecanismos implicados en la regulación de la presión arterial; y $c$ ) una banda de frecuencias altas (desde 0.15 a $0.50 \mathrm{~Hz}$ ) que parece reflejar los efectos de la actividad respiratoria (Mulder, 1980; Mulder y Mulder, 1981). Parece que el componente medio (banda en torno a 0.10 $\mathrm{Hz}$ ) es el que específicamente varía con la carga de trabajo mental. Aasman, Mulder y Mulder (1987) encontraron una disminución en la amplitud de la banda $0.10 \mathrm{~Hz}$ cuando se incrementaba la carga de procesamiento sobre la memoria de trabajo en tareas de elección forzosa y reconocimiento. Este hallazgo les llevó a sugerir que la amplitud del componente $0.10 \mathrm{~Hz}$ puede utilizarse como índice de las demandas de procesamiento controlado o esfuerzo. Vicente, Morton y Moray (1987) confirmaron el dato de Aasman y cols. (1987), y además informaron de una correlación negativa entre la amplitud $01.0 \mathrm{~Hz}$ y esfuerzo subjetivo.

Si es cierto que, tal como sugiere la hipótesis de Parasuraman, la atención declina a lo largo del tiempo durante la realización de tareas de vigilancia, entonces debería encontrarse un incremento en la amplitud del componente 0.10 $\mathrm{Hz}$. Recientemente, Carter y Beh (1989) estudiaron el cambio en la amplitud de este componente durante una tarea de vigilancia. Registraron la actividad cardiovascular mientras los sujetos hacían una tarea de vigilancia bajo distintas condiciones de ruido intermitente y silencio. Encontraron que la amplitud 0.10 $\mathrm{Hz}$ variaba según el tipo de ruido; si embargo, a pesar de que había un decremento de rendimiento significativo a lo largo de la tarea de vigilancia, no encontraron un cambio significativo en esa amplitud entre el comienzo y final de la sesión (los autores no informaron de correlaciones entre la medida fisiológica y las de rendimiento).

\section{METODO}

\section{Sujetos}

Participaron 28 estudiantes universitarios (15 mujeres y 13 hombres) con edades entre 19 y 32 años (media $=22$ ). Ninguno de ellos tenía experiencia previa 
con tareas de vigilancia. Todos informaron de visión normal o adecuadamente corregida.

\section{Diseño}

Un grupo de 20 sujetos realizó las dos tareas primarias de vigilancia (una de discriminación sucesiva y otra de discriminación simultánea) y, al mismo tiempo, la tarea secundaria, consistente en detectar un tono que aparecía entre 100-300 o entre 900-1.100 milisegundos después del comienzo de la presentación del estímulo visual. Empleamos, por lo tanto, un diseño 2 (Discriminación) $\times 2$ (posición del tono), con medidas repetidas en los dos factores. El orden de las tareas de vigilancia se contrabalanceó entre sujetos.

Otros ocho sujetos sirvieron como grupo control para las medidas fisiológicas. Estos sujetos participaron en una sesión en la que simplemente no hacían nada durante todo el tiempo que duraban las tareas de vigilancia.

\section{Aparatos.}

Todas las sesiones fueron individuales y supervisadas por un ayudante de laboratorio. El experimento se controló por ordenador. Programamos un ordenador IBM PC/XT con un monitor IBM monocromo para presentar los estímulos y registrar las respuestas. Para establecer los intervalos temporales y registrar los tiempos de reacción, empleamos una subrutina en lenguaje máquina escrita por Graves y Bradley (1987).

El electrocardiograma (ECG) se registró utilizando una derivación precordial. Los tiempos de ocurrencia de las ondas R del ECG se establecieron por un detector automático del complejo QRS, con una precisión de 1 milisegundo, y se almacenaron en una memoria para posteriores análisis con el ordenador (ver Mulder, 1988, para una discripción completa del equipo ECG).

\section{Tareas}

Las tareas de vigilancia eran similares a las empleadas por Parasuraman y Mouloua (1987). Las tareas requerían la discriminación sucesiva o simultánea de diferencias en las longitudes de pares de líneas verticales. Los estímulos se presentaban durante $150 \mathrm{mseg}$. en la pantalla del ordenador en modo gráfico $(640 \times 200$ pixels), alternativamente en el lado derecho o izquierdo a nivel del ojo. El estímulo ruido o no-señal era un par de líneas de una longitud de 39 pixels cada una separadas 38 pixels. A una distancia de visualización de $50 \mathrm{~cm}$, cada una de estas líneas subtendía un ángulo visual de $4.46^{\circ}$. En la tarea de discriminación simultánea, el estímulo señal estaba formado por una línea de 37 pixels $\left(4.24^{\circ}\right)$ y otra de 39 pixels (la línea más corta aparecía con igual probabilidad y el azar a derecha e izquierda); en la tarea de discriminación sucesiva, el estímulo señal era un par de líneas de 34 pixels de longitud $\left(3.90^{\circ}\right)$. Las diferencias en longitudes se seleccionaron en base a estudios piloto de modo que la discriminación señal/ruido, indicada por $\mathrm{P}(\mathrm{A})$, medida no paramétrica de la teoría detección de señales (Normal y Pollack, 1964), fuese aproximadamente 0.90 en ambas tareas. Los sujetos debían responder a los estímulos señal presionando la barra espaciadora en el teclado del ordenador. Cada una de las tareas duraba 30 minutos. Los estímulos se presentaban sin interrupciones a un ritmo de 30 por minuto y con una probabilidad de señal igual a 0.10 . En total, 
se presentaron 90 estímulos señal y 810 no-señales. Los 900 ensayos se dividieron posteriormente en dos bloques de 450 para el análisis de datos.

Dieciocho tonos de $1.000 \mathrm{~Hz}$ claramente audibles emitidos por el ordenador durante $150 \mathrm{mseg}$, se presentaron a través de auriculares en cada mitad de la tarea. Nueve tonos aparecían entre 100 y $300 \mathrm{mseg}$ y otros nueve entre 900 y 1.100 mseg después del comienzo de la presentación del par de líneas. Dos tonos de cada tipo se presentaban después de un estímulo visual señal y los otros siete después de un estímulo no-señal. Los sujetos tenían que responder a los tonos presionando otra tecla en el teclado del ordenador.

\section{Procedimiento}

Todos los sujetos hacían las dos tareas de vigilancia en días diferentes a la misma hora (el intervalo entre sesiones fue aproximadamente de una semana). Cada sesión duraba unos 50 minutos. A la llegada al laboratorio, el sujeto se sentaba frente al monitor a una distancia aproximada de $50 \mathrm{~cm}$ y se le colocaban los electrodos para el registro de la actividad cardiovascular. Las instrucciones destacaban que debían contestar del modo más preciso y rápido posible. Primero, los sujetos hacían dos bloques de práctica de tres minutos cada uno, y al final de cada uno se les daba información sobre su rendimiento (porcentajes de éxitos y falsas alarmas). Después del entrenamiento, se les daba un descanso de cinco minutos antes de empezar la tarea de vigilancia, y durante este período se hacía un registro fisiológico para determinar la línea base de su actividad cardiovascular. Finalizado este período, hacían la tarea sin interrupciones. Al final de la tarea, el sujeto completaba las escalas de las NASA-TLX. Finalizada la primera sesión, se les convocaba para la segunda.

Los sujetos del grupo control participaban sólo en una sesión de laboratorio, en las mismas condiciones que el grupo experimental pero sin hacer ninguna tarea.

\section{RESULTADOS}

\section{Rendimiento en las tareas primarias de vigilancia}

En el análisis de rendimiento, dividimos el tiempo total en la tarea en períodos de 15 minutos cada uno. En cada uno de estos períodos obtuvimos cuatro medidas: probabilidades de éxitos, $\mathrm{P}(\mathrm{E})$, y falsas alarmas, $\mathrm{P}(\mathrm{FA})$, tiempo de reacción para las respuestas correctas o éxitos, $\mathrm{TR}$, y $\mathrm{P}(\mathrm{A})$. La Tabla I muestra los valores medios de estas medidas en cada período de cada tarea. Hicimos un MANOVA 2 (discriminación: sucesiva, simultánea) $\times 2$ (Tiempo: primer, segundo período), con medidas repetidas en ambos factores, con cada una de las medidas.

No encontramos ningún efecto principal ni interacción entre factores en el MANOVA de los TRs. Los MANOVAs de éxitos y falsas alarmas mostraron un efecto principal del factor Tiempo $(F(1,16)=4.69 ;$ p. <.046; y $F(1,16)=7.28$; p. $<.016$, respectivamente): tanto el porcentaje de éxitos como el de falsas alarmas declinaban en el segundo período de cada tarea. El MANOVA de los valores $\mathrm{P}(\mathrm{A})$ tampoco mostró ningún efecto principal. Este resultado negativo sugiere que los decrementos en $\mathrm{P}(\mathrm{E})$ y $\mathrm{P}(\mathrm{FA})$ a lo largo del tiempo estaban terminados por cambios en el criterio de decisión, no por un decremento de la sensibilidad: el criterio de decisión se hacía más estricto con el tiempo en la tarea, disminuyendo así la proporción de respuestas «sí». 
TABLA I

Rendimiento en una tarea de discriminación sucesiva y en otra de discriminación simultánea (proporciones de éxitos y falsas alarmas, $P(A), T R$ de respuestas correctas y eficiencia), indices cardiovasculares (energia espectral media en la banda $0.1 \mathrm{~Hz}$ e IBI medio), y TRs a tonos concurrentes, en dos períodos de 15 minutos cada uno. Los niveles de línea base de cada medida cardiovascular aparecen entre paréntesis.

Discriminación

\begin{tabular}{lccccc}
\hline & \multicolumn{2}{c}{ sucesiva } & \multicolumn{2}{c}{ simultánea } \\
\cline { 2 - 3 } \cline { 5 - 6 } Rendimiento en & Bloque-1 & Bloque-2 & Bloque-1 & Bloque-2 \\
la tarea primaria & & & & \\
P (E) & & & & \\
P (FA) & .668 & .611 & .651 & .610 \\
P (A) & .012 & .005 & .008 & .005 \\
TR (mseg.) & .912 & .900 & .910 & .900 \\
P (A) VTR & 659 & 672 & 686 & 653 \\
TRs a tonos & .036 & .035 & .034 & .033 \\
Tempranos (100-300 ms) & & & & & \\
Tardíos (900-1.100 ms) & 803 & 765 & 732 & 749 \\
Indices & 538 & 506 & & 518 & 493 \\
cardiovasculares & & & & \\
Amplitud 0.1 Hz (1891) & 1463 & 1791 & 1725 & 1943 \\
IBI (ms) (610) & 704 & 720 & 727 & 734 \\
\hline
\end{tabular}

Calculamos coeficientes de correlación de Pearson entre los valores $\mathrm{P}(\mathrm{A})$ y los TRs en cada período de cada tarea. Todas las correlaciones fueron significativas y de signo negativo: -0.52 y -0.60 en los dos períodos de la tarea de discriminación simultánea, $\mathrm{y}-0.49$ y -0.48 en la de discriminación sucesiva. Por último, calculamos las razones $\mathrm{P}(\mathrm{A}) / \sqrt{ } \mathrm{TR}$ para cada período de cada tarea. Con estos nuevos datos, hicimos un MANOVA 2 (Discriminación) $\times 2$ (Tiempo). Este análisis mostró que la razón $\mathrm{P}(\mathrm{A}) / \sqrt{ } \mathrm{TR}$ decrecía significativamente durante el segundo período de ambas tareas $(F(1,16)=5.29 ;$ p. $<0.03)$. No encontramos interacción entre los dos factores. Puesto que los cambios en precisión y velocidad iban en la misma dirección (niveles más altos de precisión estaban asociados a ejecución más rápida), el decremento en $\mathrm{P}(\mathrm{A}) / \sqrt{ } \mathrm{TR}$ puede interpretarse como un cambio en la eficiencia sensorial o ritmo de procesameinto de información.

\section{Carga de trabajo subjetiva}

Las evaluaciones ponderadas en la eslaca de esfuerzo y el nivel total de carga de trabajo en la NASA-TLX en ambas tareas se compararon con una prueba $t$ de Student. No encontramos ninguna diferencia significativa.

\section{Rendimiento en la tarea secundaria}

La Tabla I muestra también los TRs a los tonos. Estos datos fueron analizados en un MANOVA 2 (discriminación) $\times 2$ (Tiempo) $\times 2$ (Posición del tono), 
con medidas repetidas en los tres factores. Sólo encontramos un efecto principal significativo del factor Posición $(F(1,16)=41.75$; P. <.001): los sujetos respondían más rápido a los tonos tardíos (900-1.100 mseg. después del comienzo del estímulo visual) que a los tonos tempranos (100-300 mseg.) (510 vs $760 \mathrm{mseg}$.). Puesto que el efecto del factor tiempo no fue significativo, el decremento observado en $\mathrm{P}(\mathrm{A}) / \sqrt{ } \mathrm{TR}$ en la tarea primaria no estaba, pues, asociado con un cambio similar en rendimiento en la tarea secundaria. Este hallazgo no parece consistente con la hipótesis de recursos atencionales del decremento de vigilancia.

\section{Medidas de actividad cardiovascular}

En el análisis de los datos fisiológicos no incluimos a cuatro sujetos del grupo experimental debido al mal funcionamiento del equipo durante los respectivos registros. No rechazamos ningún sujeto del grupo control. La sesión completa la dividimos en siete períodos de cinco minutos, el primero correspondiente a la línea base y los seis restantes a la realización de la tarea. Calculamos las medias del IBI y amplitud $0.1 \mathrm{~Hz}$ utilizando el programa CARSPAN (ver Mulder, 1988, para una descripción de este programa). Debido a las grandes diferencias individuales, estos valores medios se convirtieron a porcentaje de cambio en relación a la línea base.

En primer lugar, con objeto de comparar más fácilmente los datos fisiológicos con las medidas conductuales, calculamos los valores medios de IBI y amplitud $0.1 \mathrm{~Hz}$ para cada período de 15 minutos (ver Tabla I). Hicimos un MANOVA 2 (Discriminación) $\times 2$ (Tiempo para cada una de estas medidas fisiológicas. No encontramos ningún efecto principal significativo en el análisis de IBIs; en el análisis de las amplitudes $0.1 \mathrm{~Hz}$ encontramos un efecto principal significativo del factor Tiempo $(\mathrm{F}(1.12)=8.67$, p. <0.0015): esta amplitud aumentaba en la segunda mitad de la tarea. La interacción entre factores no fue significativa en ninguno de los análisis. En general, estos resultados sugieren que la carga de memoria en las dos tareas de vigilancia no difería lo suficiente como para reflejarse en las medidas cardiovasculares. Esta conclusión se evaluó también comparando las medidas de línea base con los cinco primeros minutos de cada tarea en un MANOVA 2 (Discriminación) $\times 2$ (Tiempo: línea base, primeros cinco minutos). Para los IBIs, no encontramos ningún efecto principal significativo ni interacción; para la amplitud $0.1 \mathrm{~Hz}$, encontramos un efecto principal significativo del factor Tiempo $(F(1,12)=10.39$, p. $<0.007)$, pero no del factor Discriminación: la amplitud decrecía significativamente por igual en ambas tareas en los cinco primeros minutos.

El análisis de los datos del grupo control no mostró ningún efecto significativo del Tiempo para ninguna de las dos medidas. Así pues, el incremento de la amplitud $0.1 \mathrm{~Hz}$ durante la segunda mitad de la tarea no podía deberse simplemente a la situación experimental per se.

Finalmente, hicimos comparaciones entre el grupo control y ambas condiciones de tarea para cada índice fisiológico medido en tres períodos: $0-5 \mathrm{minu}$ tos (primeros cinco minutos), 0-15 min. (primera mitad) y $15-30 \mathrm{~min}$. (segunda mitad). Sólo encontramos una diferencia significativa entre la condición control y la de discriminación sucesiva en la amplitud $0.10 \mathrm{~Hz}$ durante los primeros cinco minutos $(F(1,22)=6.06$, p. $<.02)$ : la amplitud en este período era más baja en el grupo de sujetos que hacía la tarea de discriminación sucesiva que en el grupo de sujetos control. En la condición de discriminación simultánea, esta diferencia no fue significativa. 


\section{4}

Las figuras I y II representan los valores medios de IBI y amplitud $0.10 \mathrm{~Hz}$, respectivamente, en función del tiempo (5,15 y 30 minutos), para cada condición.

\section{FIGURA I}

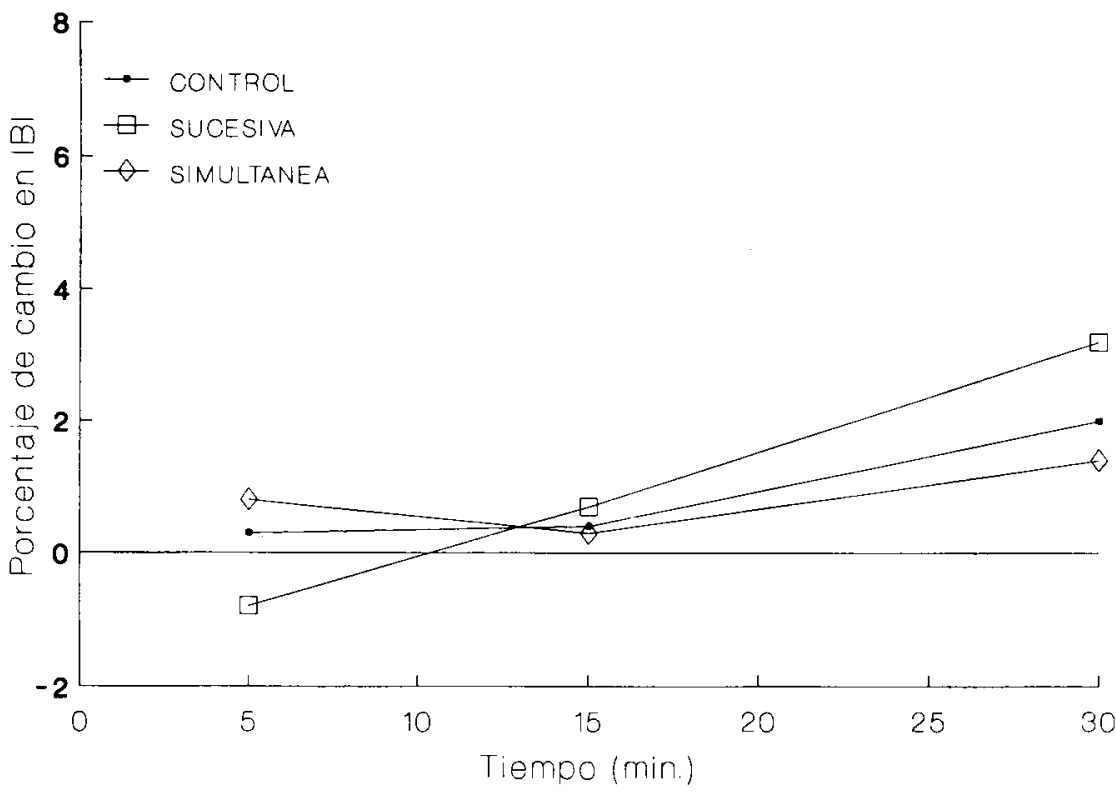

Correlatos del decremento de vigilancia. Calculamos las correlaciones de Pearson entre las diferentes variables dependientes de nuestro experimento y el decremento de vigilancia en cada tarea. El decremento de vigilancia se midió por la diferencia en el nivel de eficiencia o $\mathrm{P}(\mathrm{A}) / \sqrt{ } \mathrm{TR}$ entre la primera y segunda parte de cada tarea (un valor positivo indica decremento). Como posible correlatos incluimos las siguientes medidas: NASATLX, TRs a tonos en cada mitad de la tarea, medidas fisiológicas (IBI y amplitud $0.1 \mathrm{~Hz}$ ) en cada mitad de la tarea y en los cinco primeros minutos, y el cambio en cada una de estas medidas entre la primer y segunda mitad de la tarea. Las correlaciones aparecen en la Tabla II. El correlato más importante del decremento de vigilancia en ambas tareas resultó ser la amplitud $0.1 \mathrm{~Hz}$ en los cinco primeros minutos: altas amplitudes estaban asociadas a niveles altos de decremento. Si esta amplitud está inversamente relacionada con las demandas de procesamiento controlado (Aasman y cols., 1987; Vicente y cols., 1987), este hallazgo sugiere que el decremento de vigilancia en ambas tareas estaba asociado a niveles bajos de procesamiento controlado o esfuerzo al comienzo de la tarea. Calculamos, pues, las ecuaciones de regresión simple para determinar qué parte de la varianza en el decremento de vigilancia en cada tarea podría explicarse por la amplitud $0.1 \mathrm{~Hz}$ durante los cinco primeros minutos. En la condición de discriminación sucesiva, la ecuación de regresión daba cuenta del $44.9 \%$ de la varianza $(F(1,15)=13.67, p<.003)$, mientras que en la condición de discriminación simultánea, la ecuación de regresión explicaba el $26.1 \%$ de la varianza $(F(1,15)=4.93$, p. $<0.43)$. Así pues, 
FIGURA II

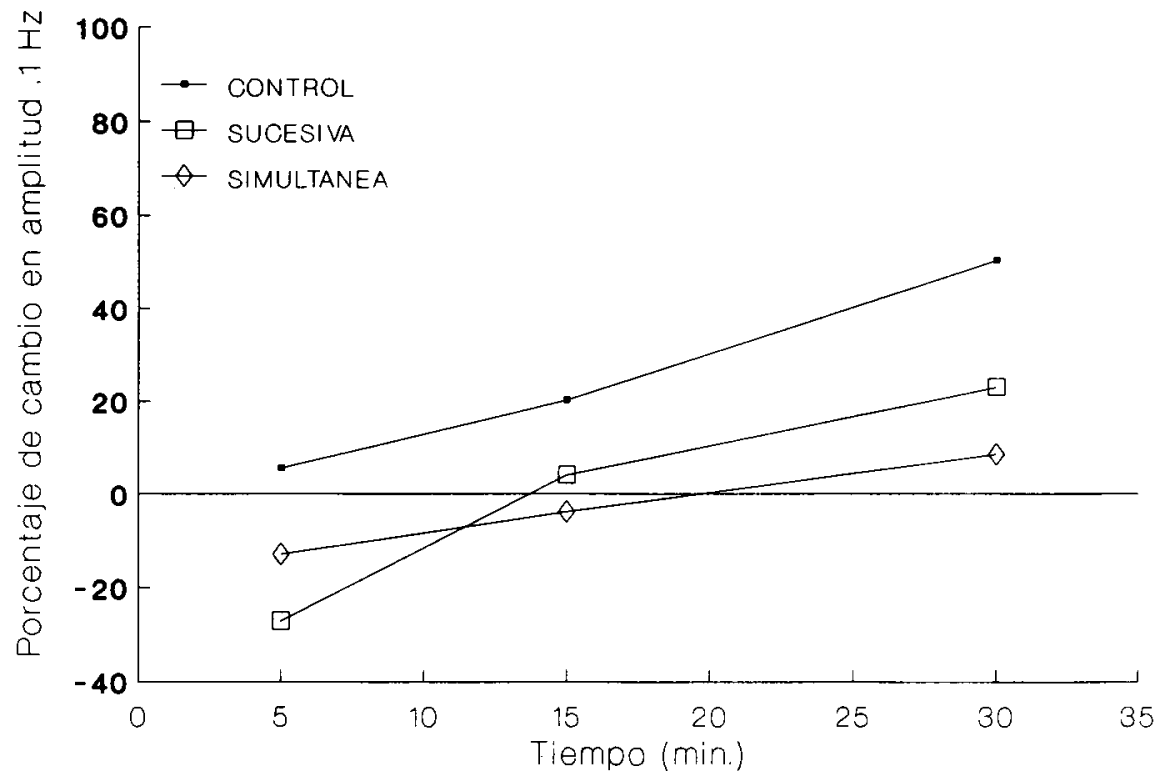

la amplitud del componente $0.1 \mathrm{~Hz}$ al comienzo de la tarea era un predictor importante del decremento de vigilancia.

\section{TABLA II}

Correlaciones de Pearson entre medidas de carga de trabajo y decremento de vigilancia, indicado por la diferencia en el nivel de eficiencia sensorial $(P(A) / \sqrt{T R})$ entre el primer y segundo períodos de cada tarea (valores positivos indican decremento o declive en eficiencia)

\begin{tabular}{|c|c|c|}
\hline & Disc & ación \\
\hline & Sucesiva & Simultánea \\
\hline Medidas subjetivas & & \\
\hline Esfuerzo & .35 & .15 \\
\hline Carga total & .09 & .10 \\
\hline Medidas en la tarea secundaria & & \\
\hline Tonos tempranos $(0-15 \mathrm{~min})$ & $.44^{*}$ & -.09 \\
\hline Tonos tempranos $(15-30 \mathrm{~min})$ & .07 & -.29 \\
\hline Tonos tardíos $(0-15 \mathrm{~min})$ & $.51^{*}$ & -.07 \\
\hline Tonos tardíos $(15.30 \mathrm{~min})$ & .21 & -.37 \\
\hline Indices cardiovasculares & & \\
\hline $0.10 \mathrm{~Hz}(0-5 \mathrm{~min})$ & $.70^{* * *}$ & $.51^{*}$ \\
\hline $0.10 \mathrm{~Hz}(0-15 \mathrm{~min})$ & .35 & $.63 * *$ \\
\hline $0.10 \mathrm{~Hz}(15-30 \mathrm{~min})$ & .21 & $.60^{\star}$ \\
\hline Cambio en $0.10 \mathrm{~Hz}$ & .24 & $-.46^{*}$ \\
\hline IBI $(0-5 \mathrm{~min})$ & .03 & .21 \\
\hline IBI (0-15 min) & -.05 & .15 \\
\hline IBI $(15-30 \mathrm{~min})$ & -.29 & -.15 \\
\hline Cambio en IBI & .32 & .39 \\
\hline
\end{tabular}

${ }^{*}$ p. $<.05$ (dos colas); ${ }^{* *}$ p. $<.01 ;{ }^{* * *}$ p. $<.001$. 
También encontramos una correlación significativa positiva entre los TRs a tonos tardíos en la primera parte de la tarea y del decremento de vigilancia, pero sólo en la condición de discriminación sucesiva, no en la de discriminación simultánea: TRs más largos en esta condición estaban asociados a niveles más altos de decremento. Ninguna de las correlaciones del decremento de vigilancia con los IBs y los índices de la NASA-TLX resultaron significativas.

\section{DISCUSION}

El objetivo del experimento era probar la hipótesis de que el decremento de sensibilidad en tareas de vigilancia está asociado a niveles altos de carga de trabajo mental y disminución de recursos atencionales (Parasuraman, 1985). Según esta hipótesis, el decremento de vigilancia se debe a que las tareas de atención sostenida demandan, a partir de cierto momento en su realización, más recursos atencionales de los disponibles. Comparamos dos tareas que diferían en carga de memoria o tipo de discriminación, sucesiva o simultánea, en varios grupos de medidas: medidas conductuales, carga subjetiva de trabajo y medidas de actividad cardiovascular. El decremento de sensibilidad en ambas tareas no fue significativo, pero observamos un declive significativo en ambas condiciones en el nivel de eficiencia o ritmo de procesamiento de información, indicado éste por la razón entre el nivel de precisión y la velocidad. No encontramos ninguna diferencia significativa en rendimiento entre las dos tareas.

Estos resultados difieren de los obtenidos por Parasuraman y Mouloua (1987), quienes encontraron decremento de sensibilidad en tareas de discriminación sucesiva y simultánea de diferencias en las longitudes de líneas. Parasuraman y Mouloua utilizaron tres niveles de discriminación señal/no-señal: alto $(\mathrm{P}(\mathrm{A})=0.96$ en ambas tareas), moderado (0.93 en la de discriminación sucesiva y 0.92 en la de discriminación simultánea) y bajo (0.90 en la de discriminación sucesiva y 0.93 en la de discriminación simultánea). En nuestro experimento, el nivel de discriminabilidad fue 0.91 en ambas tareas y, por lo tanto, es equiparable al nivel de discriminabilidad bajo empleado por Parasuraman y Mouloua. La discrepancia entre nuestros resultados y los de estos investigadores quizás pue$\mathrm{da}$ explicarse por el diferente ritmo de estimulación empleado en ambos experimentos: 30 estímulos/minuto en nuestro experimento y 40 estímulos/minuto en el de Parasuraman y Mouloua.

Dos grupos de datos no parecen apoyar la teoría de Parasuraman del decremento de vigilancia. Primero, una predicción de esta teoría es que el decremento de rendimiento en tareas de vigilancia debería covariar con el esfuerzo subjetivo; sin embargo, no encontramos ninguna correlación entre el esfuerzo subjetivo, medido por la NASA-TLX, y el nivel de decremento. Este resultado negativo confirma un hallazgo reciente de Koelega y cols. (1989), quienes tampoco encontraron correlaciones entre esfuerzo subjetivo y rendimiento en distintas tareas de vigilancia. Una limitación de las técnicas subjetivas para medir la carga de trabajo o esfuerzo es su dependencia de la memoria, dado que estas técnicas requieren que el sujeto recuerde la cantidad de trabajo mental durante la tarea. Así, pudiera ser que las evaluaciones varien en función del intervalo entre la ejecución de la tarea y el informe subjetivo. En condiciones de vigilancia, los sujetos no pueden dar sus informes durante la ejecución de la tarea, puesto que este procedimiento produciría interrupciones que prevendrían el decremen- 
to de rendimiento, y, por lo tanto, necesariamente deben existir intervalos largos entre el comienzo de la tarea y el momento en que da el informe. Este intervalo podría hacer que el sujeto utilizase la memoria de un modo diferente y evaluase así distintos aspectos de su ejecución.

Segundo, otro dato que no parece apoyar la teoría de Parasuraman es que el rendimiento en la tarea secundaria fue estable durante toda la sesión, a pesar de que disminuía la eficiencia de procesamiento en la tarea de vigilancia primaria. Una interpretación posible de estos datos es que la ejecución en la tarea secundaria podría haber sido automática y no depender de los recursos atencionales del sujeto (Shiffrin y Schneider, 1977). Esta interpretación, sin embargo, no puede aplicarse en nuestro experimento, puesto que los sujetos tenían que responder a un número muy reducido de tonos (18 en cada período de la tarea) y esto, evidentemente, no facilita la automatización. Otra posibilidad es que la carga total de trabajo impuesta por la tarea de vigilancia primaria no hubiera excedido la capacidad del sujeto (o cantidad total de recursos disponibles), con lo cual, la tarea secundaria de detección auditiva no sería sensible a variaciones en el nivel de ejecución de vigilancia. Pero, si esta interpretación fuese correcta, entonces el decremento en eficiencia que observamos en nuestro experimento no podría explicarse por la teoría de Parasuraman $(1979,1985)$ que, recordemos, se basa en una limitación de los recursos atencionales. Además, el hecho de que los TRs fuesen más altos a los tonos presentados 100-300 mseg. que a los presentados $900-1.100$ mseg. después del comienzo del estímulo visual, sugiere que las respuestas a los tonos tempranos podrían basarse en el mismo conjunto de recursos que el procesamiento de los estímulos visuales. Tomando en cuenta esta diferencia y que el TR medio a líneas fue aproximadamente 667 mseg., podemos suponer que, cuando se presentaban los tonos tempranos, el sujeto todavía no había tomado una decisión sobre si el estímulo visual era o no señal y, consecuentemente, la reacción al tono se retrasaba; por el contrario, cuando se presentaba un tono tardío, el sujeto ya había tomado una decisión acerca del estímulo visual y no tenía que retrasar su respuesta a ese tono.

Los datos referentes a la actividad cardiovascular durante las tareas de vigilancia tampoco parecen fáciles de interpretar desde la teoría de Parasuraman. Tal como se esperaba, la amplitud $0.1 \mathrm{~Hz}$ se incrementó en la segunda mitad de ambas tareas, mientras que permanecía estable en la condición control. Este hallazgo parece consistente con la hipótesis de Parasuraman. De acuerdo con hallazgos previos, el cambio en la banda media indicaría que los sujetos no podían mantener su esfuerzo durante toda la tarea, y puesto que observamos un decremento significativo en rendimiento, teníamos que observar un incremento también significativo en la amplitud de esta banda media, reflejando que los sujetos no podían mantener su atención en la tarea. Sin embargo, mientras que la amplitud $0.1 \mathrm{~Hz}$ y la eficacia cambiaban durante la sesión de vigilancia, no encontramos ninguna correlación significativa entre estos cambios.

El principal hallazgo del experimento fue que la amplitud de la banda media al comienzo de la tarea de vigilancia era un predictor significativo del decremento de rendimiento. Encontramos correlaciones entre el declive en eficiencia $[\mathrm{P}(\mathrm{A}) / \sqrt{\mathrm{TR}}]$ y la amplitud $0.1 \mathrm{~Hz}$ durante los cinco primeros minutos de cada tarea. Si esta amplitud es un índice fisiológico fiable de atención, entonces, nuestro resultado sugiere que un procesamiento con esfuerzo al comienzo de la tarea previene un declive en rendimiento (Koelega y cols., 1989). Este resultado podría parecer, en principio, incongruente con la hipótesis de Parasuraman. Según 


\section{8}

esta hipótesis, los sujetos que prestan mucha atención al comienzo de la sesión encontrarían más difícil mantenerla hacia el final y, consecuentemente, deberían mostrar un mayor decremento de rendimiento. Sin embargo, cabe otra interpretación de la relación entre la amplitud $0.1 \mathrm{~Hz}$ y el decremento de vigilancia. La interpetación alternativa es que los sujetos aprendían a realizar «mejor» la tarea cuando prestaban mucha atención al comienzo de la misma. Este aprendizaje podría consistir en el desarrollo de estrategias que redujesen las demandas cognitivas de la tarea y previniesen el decremento.

Por último, no encontramos ninguna relación entre la media del ritmo cardíaco (que es la inversa del IBI) y las medidas de rendimiento en las tareas de vigilancia, $\mathrm{y}$, lo que quizás sea más importante, los mismos cambios en el ritmo cardíaco se encontraron cuando los sujetos no hacían ninguna tarea (grupo control). Experimentos previos indican que no existe un patrón claro del ritmo cardíaco en tareas de vigilancia. Mientras que algunos autores han encontrado un declive progresivo en el ritmo cardíaco (p.e. Griew y cols., 1963; Thackray y cols., 1974), otros han encontrado un incremento (p.e. Carter y Beh, 1989). Pudiera ser que el ritmo cardíaco no fuese un correlato fisiológico fiable del decremento de vigilancia, sino de la situación experimental específica.

En resumen, los datos de este experimento no se ajustan bien a las hipótesis de Parasuraman $(1979,1985)$, según la cual, el decremento de vigilancia se debe a un declive en la capacidad atencional del sujeto conforme avanza la sesión del trabajo. Es posible que el modo en que los sujetos «encaran» la tarea sea un predictor importante del decremento de vigilancia. Sin embargo, nuestro experimento no fue diseñado específicamente para probar esta hipótesis. Parece necesario hacer otros experimentos para probar cómo el decremento de vigilancia se ve afectado por la estrategia o actitud con la que el sujeto afronta la tarea, y que define el tipo y cantidad de recursos atencionales que está dispuesto a invertir de acuerdo con un criterio óptimo de rendimiento establecido por él.

\section{Referencias}

Aasman, J.; Mulder, G. y Mulder, L. J. M. (1987): Operator Effort and the Measurement of Heart-Rate Variability. Human Factors, 29, 161-170.

Carter, N. L. y Beh, H. C. (1989): The Effect of Intermittent Noise on Cardiovascular Functioning During Vigilance Task Performance. Psychophisiology, 26, 548-559.

Davies, D. R. y Parasuraman, R. (1982): The Psychology of vigilance. Londres: Academic Press.

Fleishman, E. A. (1972). On the relation between abilties, learning and human performance. American Psychologist, 27, 1017-1032.

Fleishman, E. A. y Quaintance, M. K. (1984). Toward a taxonomy of human performance. American Psychologist, 30, 1127-1149.

Fisk, A. D. Y SCHNEIDER, W. (1981): Control and automatic processing during tasks requiring sustained attention: A new approach to vigilance. Human Factors, 23, 737-750.

Hart, S. G. y Staveland, L. E. (1989): Development of Nasa-TLX (Task Load Index): Results of Empirical and Theoretical Research. En P. A. Hancock y N. Meshkati (comps.), Human Mental Workload. Amsterdam: North-Holland.

Koelega, H. S., Brinkman, J., Hendriks, L. y Verbaten, M. N. (1989): Processing Demands, Effort, and Individual Differences in Four Different Vigilance Tasks. Human Factors, $31,45-62$.

Mulder, G. (1980): The Heart of Mental Effort. Tesis Doctoral. Universidad de Groningen. Mulder, G. y Mulder, L. J. M. (1981): Information Processing and Cardiovascular Control. Psychophysiology, 14, 392-402. 
Mulder, L. J. M. (1988): Assesment of Cardiovascular Activity by means of Spectral Analysis. Tesis Doctoral. Universidad de Groningen.

Nuechterlein, K., Parasuramann, R. y Jiang, Q. (1983): Visual sustained attention: Image degradation produces rapid sensivity decrement over time. Science, 220, 327-329.

Parasuraman, R. (1979): Memory load and event rate control sensitivity decrements in sustained attention. Science, 205, 924-927.

Parasuraman, R. (1985): Sustained attention: A multifactorial approach. En M. I. Posner y O.S.M. Marin (comps.), Attention and performance XI. Hillsdale, NJ: Erlbaum.

Parasuraman, R. y Davies, D. R. (1977): A taxonomic analysis of vigilance performance. En R.R. Mackie (comp.), Vigilance: Theory, operational performance, and physiological correlates. Nueva York: Plenum Press.

Parasuraman, R. y Mouloua, M. (1987): Interaction of signal discriminability and task type in vigilance decrement. Perception y Psychophysics, 41, 17-22.

SHIfFRIN, R. M. Y SCHNEIDER, W. (1977): Controlled and automatic human information processing: I. Detection, search, and attention. Psychological Review, 84, 1-66.

ThackRay, Jones y Tocmsione, (1974).

VICENTE, K. J., Thornton, D. C. y Moray, N. (1987): Spectral Analysis of Sinus Arrhythmia: A Measure of Mental Effort. Human Factors, 29, 171-182.

Wier, Curry y Faustra (1984) 